\title{
The sensitivity of modulation fidelity on PA envelope variation in OFDM transmitter systems
}

\author{
Yiming Lei ${ }^{* \dagger}$, Mingke Dong ${ }^{\dagger}$ and Ye Jin
}

\begin{abstract}
Intermodulation distortion (IMD) caused by nonlinear power amplifier (PA) is a major weakness in orthogonal frequency-division multiplexing (OFDM) transmitter systems, and most behavioral studies of IMD are based on specific example PAs. In recent papers, PA percentage linearization (PL), a general measurement for PA envelope variation, is investigated for its influence on system performance. Meanwhile, PL still somehow depends on specific example PA, and it mostly considers the PA saturation point (SP) variation. In this paper, a comparison analysis is presented to investigate the system performance when both SP and nonlinearity onset point (NOP) variations are considered. The simulations show that how the PA envelope variation can be represented by the signal impairment over IBO and $\mathrm{OBO}$ domains, respectively. It is interesting to find that although SP attracts much concern in current studies, NOP actually has a clear influence on modulation fidelity of nonlinear OFDM transmitter systems. Moreover, it is observed that when input power gets close to saturation range, the PAs having relatively higher nonlinearity show relatively lower distortion level over the input power domain. These interesting simulation observations above are analytically explained based on a mixed time domain and statistical analysis of IMD mechanism. An example IEEE 802.11a OFDM signal is driving through five example solid state PAs, and the system performance is measured by error vector magnitude (EVM) and adjacent channel power ratio (ACPR).
\end{abstract}

Keywords: Intermodulation distortion; OFDM; Nonlinear power amplifier

\section{Introduction}

Orthogonal frequency-division multiplexing (OFDM)modulated signals are widely used in wireless communications systems for their advantage of high spectrum efficiency [1,2]. However, OFDM signals have a nature of high peak to average power ration (PAPR), since they consist of lots of independent input subcarriers. This high PAPR nature makes OFDM transmitter systems very sensitive to the nonlinearity of RF power amplifiers (PAs) integrated within transmitters, and significant nonlinear distortion generated in the amplification process is a major weakness of OFDM signal systems [3,4]. The nonlinear distortion can be evaluated by the modulation

*Correspondence: leiym@pku.edu.cn

†Equal contributors

State Key Laboratory of Advanced Optical Communication Systems and Networks, School of Electronic Engineering and Computer Science, Peking University, Beijing 100871, China fidelity over wanted subcarriers and the out-of-band spectral regrowth, and they can be quantitatively expressed by error vector magnitude (EVM) and adjacent channel power ration (ACPR), respectively [5].

For the designers of OFDM transmitter systems, the influence of PA envelope character on system modulation fidelity is a fundamental concern, and it attracts lots of studies [6-9]. However, most relevant studies are based on a specific example PA, and the influence of PA envelope variation on system performance is not well explored yet. In recent papers $[10,11]$, the PA envelopes are characterized by percentage linearization (PL) determined by PA saturation point (SP), and the influence of PL on system modulation fidelity is investigated. Meanwhile, the influence of PA envelope is not investigated yet as both SP and nonlinear onset point (NOP) variations are considered. Thus, a couple of questions can be asked here. What is the influence of PA envelope on modulation fidelity performance of nonlinear OFDM transmitter systems when

\section{望 Springer}

(c) 2014 Lei et al: licensee Springer. This is an Open Access article distributed under the terms of the Creative Commons Attribution License (http://creativecommons.org/licenses/by/2.0), which permits unrestricted use, distribution, and reproduction in any medium, provided the original work is properly credited. 
both NOP and SP variations are considered? Can the simulation observations be explained analytically? This paper presents our efforts to answer these questions.

In this paper, the characteristic of PAs is considered as some kind of degradations from that of the ideal PA, i.e., a fully linearized hard limiter. This degradation is characterized by both NOP and SP defined in $[10,11]$. Totally, five solid-state PA (SSPAs) envelopes with various settings of NOP and SP are designed, where the AM/PM conversion of these PA envelopes are neglected as in [10,12], and these example PAs are modeled by Bessel-Fourier (BF) behavioral model [13,14]. A 16QAM-modulated IEEE 802.11a OFDM signal is driven through these designed PAs. The intermodulation distortion (IMD) of output signal of example PAs is measured by EVM and ACPR, and the obtained EVM and ACPR results are displayed over input backoff (IBO) and output backoff (OBO) domains, respectively. This allows us to see how the variations of PA envelopes are represented through their associated $E V M / A C P R$ results. An interesting outcome is that when these example PAs are driven close to their saturation ranges, PAs having relatively higher nonlinearity show relatively lower distortion level over IBO domain. On the contrary, PAs having relatively higher nonlinearity show relatively higher distortion level over the whole OBO domain. Another interesting outcome observed from the comparative experiment is that NOP is not much concerned in the characterization of PA envelope in current studies, but the system performance actually is more sensitive to the variation of NOP than to that of SP. These simulation observations are analytically discussed by using a recent mixed time-domain and statistical analysis approach of IMD $[15,16]$. It can be seen that when input power increases, the weights of high-order terms in PA Bessel-Fourier model become comparable with those of dominated low-order terms. Therefore, the IMD of a PA envelope becomes more sensitive to the input power as it has more high-order model terms. This finding explains the simulation observations.

Section 2 presents the designed example PA envelopes. Section 3 presents the BF PA envelope model and the mixed time-domain and statistical analysis approach of IMD. The simulation observations and associated discussions are presented in Section 4, and this is followed by the conclusion in Section 5.

\section{PA envelopes having various NOPs and SPs}

In simulation-based analysis of nonlinear OFDM transmitter systems, the single SSPA transfer characteristic used can vary from device to device. Without comparative analysis of multiple-PA-based simulation experiment, valuable outcomes can be missed. In this paper, several PA envelopes having various envelope characters are designed, and the design scheme is presented below.
As in [13], generally, the input-output map of an amplification transfer system can be represented by the following nonlinear differential equation:

$$
F\left[s_{\mathrm{o}}(t), \frac{d s_{\mathrm{o}}(t)}{d t}, \ldots, \frac{d^{p} s_{\mathrm{o}}(t)}{d t^{p}}, s_{\mathrm{i}}(t), \frac{d s_{\mathrm{i}}(t)}{d t}, \ldots, \frac{d^{r} s_{\mathrm{i}}(t)}{d t^{r}}\right]=0
$$

where $s_{\mathrm{o}}(t)$ and $s_{\mathrm{i}}(t)$ are the output and input signals, respectively. In PA transmitters, the fading memory effects of transfer character is related to the input signal only. As in $[9,10,13,17,18]$, memoryless nonlinearity dominates PA transfer character, and it can be characterized by memoryless PA envelope character, i.e.,

$$
s_{\mathrm{o}}(t)=g\left[A_{\mathrm{e}}(t)\right] \cdot e^{j\left[\Phi\left(A_{\mathrm{e}}(t)\right)\right]} \cdot \frac{s_{\mathrm{i}}(t)}{A_{\mathrm{e}}(t)}
$$

where $g[$.$] and \Phi[$.$] denote PA AM/AM and AM/PM con-$ version, respectively; and $A_{\mathrm{e}}(t)$ is the envelope amplitude of input signal. Since AM/PM conversion of SSPAs does not have strong variation [13], $\Phi[$.$] of example PAs is set$ as zero.

As in $[10,11]$, PL is developed for the characterization of PA envelope, which depends on the SP variation. In this paper, five PA envelopes are presented with independent SP and NOP variation applied. The concepts of NOP, SP, and the characteristic's ideal saturation point (CISP) are shown in Figure 1. The five example PA envelopes, i.e., $L_{0}$ to $L_{4}$, are designed to have various NOP and SP degradation referring to $L_{0}$, i.e., their NOPs and SPs depart from CISP. A smooth envelope characteristic is designed to connect NOP and SP when they depart from CISP, and the derivative function of designed PA envelope smoothly decreases from 1 to 0 when the input power sweeps from NOP to SP. In this paper, the distance of NOP and SP to CISP, written as $D_{\text {NOP-CISP }}$ and $D_{\text {SP-CISP, }}$ is measured along the asymptotic ideal linearity character (AILC) line and asymptotic output saturation power (AOSP) line, respectively. Table 1 shows the $D_{\text {NOP-CISP }}$ and $D_{\text {SP-CISP }}$ of all five example PAs, and the percentage linearization area $\left(\mathrm{PL}_{\mathrm{A}}\right)$, defined in $[10,11]$, of all designed PAs, is presented in Table 1 as well. The five example PA envelopes are presented in Figure 2, and their input and output are normalized to those of CISP.

\section{IMD analysis in nonlinear OFDM transmitter systems}

The input OFDM signal including $N$ subcarriers, denoted as $s_{\mathrm{i}}(t)$ here, can be written as

$$
s_{\mathrm{i}}(t)=\sum_{l=1}^{N}\left\{A_{l}(t) \cdot e^{j\left[2 \pi f_{l} t+\varphi_{l}(t)\right]}\right\}
$$




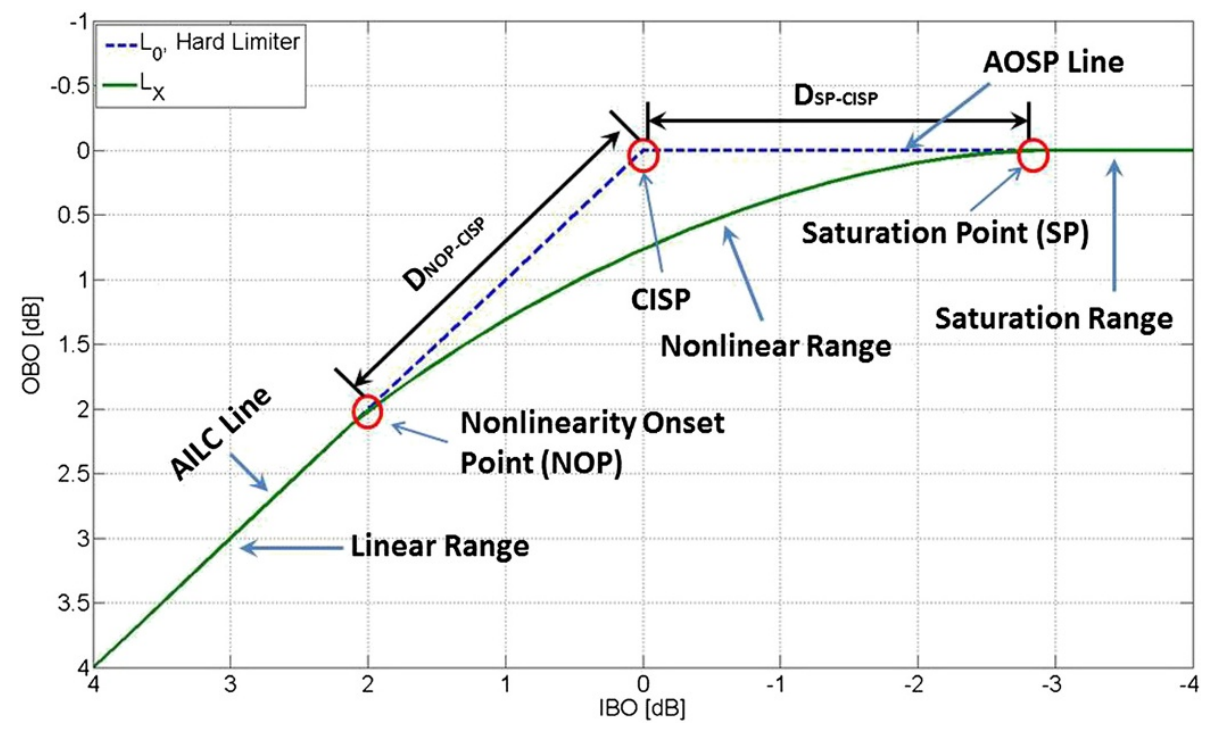

Figure 1 The characters of a typical SSPA and related concepts. The definitions of NOP, SP, and CISP are marked.

where $A_{l}(t) \varphi_{l}(t)$ and $f_{l}$ denote the amplitude, phase, and frequency of the $l$ th subcarrier. In this paper, BesselFourier behavioral model is selected for the example PA envelopes for its unique advantage when dealing with OFDM-like multi-carrier signals [13-15]. Using BesselFourier model, the zonal band PA output signal, $s_{0}(t)$, can be written as

$$
\begin{aligned}
s_{0}(t)= & \sum_{k=1}^{L} b_{k} \quad \sum_{\substack{1 \\
n_{1}, \ldots n_{N}=-\infty \\
\sum n_{l}=1}}^{\infty} \\
& \times\left\{\prod_{l=1}^{N}\left[J_{n_{l}}\left(\frac{2 \pi}{D_{\bmod }} k A_{l}(t)\right)\right] \cdot e^{j \sum_{l=1}^{N} n_{l}\left[2 \pi f_{l} t+\varphi_{l}(t)\right]}\right\}
\end{aligned}
$$

where $D_{\text {mod }}$ is the model dynamic range [18]; $J_{n_{l}}$ is the $n_{l}^{\text {th }}$ order Bessel function of the first kind; $b_{k}$ is the $k$ th coefficient of an $L$ th order Bessel-Fourier model. As in $[13,14], s_{0}(t)$ includes fundamental carriers and

Table 1 Characters of five example PA envelopes

\begin{tabular}{llc}
\hline PA & Envelope characters & $\mathbf{P L}_{\mathbf{A}}(\%)$ \\
\hline$L_{0}$ & $D_{\text {NOP-CISP }}=0 \mathrm{~dB}=D_{\text {SP-CISP }}$ & 100 \\
$L_{1}$ & $D_{\text {NOP-CISP }}=3 \mathrm{~dB}=D_{\text {SP-CISP }}$ & 75 \\
$L_{2}$ & $3 \mathrm{~dB}=D_{\text {NOP-CISP }}<D_{\text {SP-CISP }}=6 \mathrm{~dB}$ & 50 \\
$L_{3}$ & $6 \mathrm{~dB}=D_{\text {NOP-CISP }}>D_{\text {SP-CISP }}=3 \mathrm{~dB}$ & 50 \\
$L_{4}$ & $D_{\text {NOP-CISP }}=6 \mathrm{~dB}=D_{\text {SP-CISP }}$ & 0 \\
\hline
\end{tabular}

zonal band intermodulation products (IMPs), and each of them maps to a unique realization of the parameter set $\left[n_{1}, n_{2}, \ldots, n_{N}\right]$. The fundamental carriers IMPs can be distinguished by the condition of $\sum\left|n_{l}\right|=\lambda$, where $\lambda=1$ indicates fundamental carriers and other values of $\lambda(\lambda \geq 3)$ indicates the $\lambda$ th order IMPs.

Equation 4 requires an unacceptable computation cost in IMD simulation, and it can be simplified. As derived in $[15,16]$, the power level of individual fundamental carrier or IMP mapping to $\left[n_{1}, \ldots, n_{N}\right]$, denoted as $P_{\left[n_{1}, \ldots n_{N}\right]}$, can be written as

$$
P_{\left[n_{1}, \ldots n_{N}\right]}\left(\sigma^{2}\right)=\left|\sum_{k=1}^{K}\left\{b_{k} \cdot \prod_{l=1}^{N} J_{n_{l}}\left(\frac{2 \pi k}{D_{\bmod }} \sqrt{\frac{\sigma^{2}}{N}}\right)\right\}\right|^{2}
$$

where $\sigma^{2}$ denotes the power of input OFDM signal. Thus, as in [15], the power spectra of fundamental carriers and $\operatorname{IMD}$, denoted as $\operatorname{PS}_{\text {fund }}\left(f, \sigma^{2}\right)$ and $\operatorname{PS}_{\mathrm{IMD}}\left(f, \sigma^{2}\right)$, can be written as

$$
\begin{aligned}
\operatorname{PS}_{\text {fund }}\left(f, \sigma^{2}\right)= & \left(\sum_{l=1}^{N} \delta\left(f-f_{l}\right)\right) \cdot \mid \sum_{k=1}^{L} b_{k} \cdot J_{1}\left(\frac{2 \pi k}{D_{\bmod }} \sqrt{\frac{\sigma^{2}}{N}}\right) \\
& \times\left.\left[J_{0}\left(\frac{2 \pi k}{D_{\bmod }} \sqrt{\frac{\sigma^{2}}{N}}\right)\right]^{N-1}\right|^{2}
\end{aligned}
$$




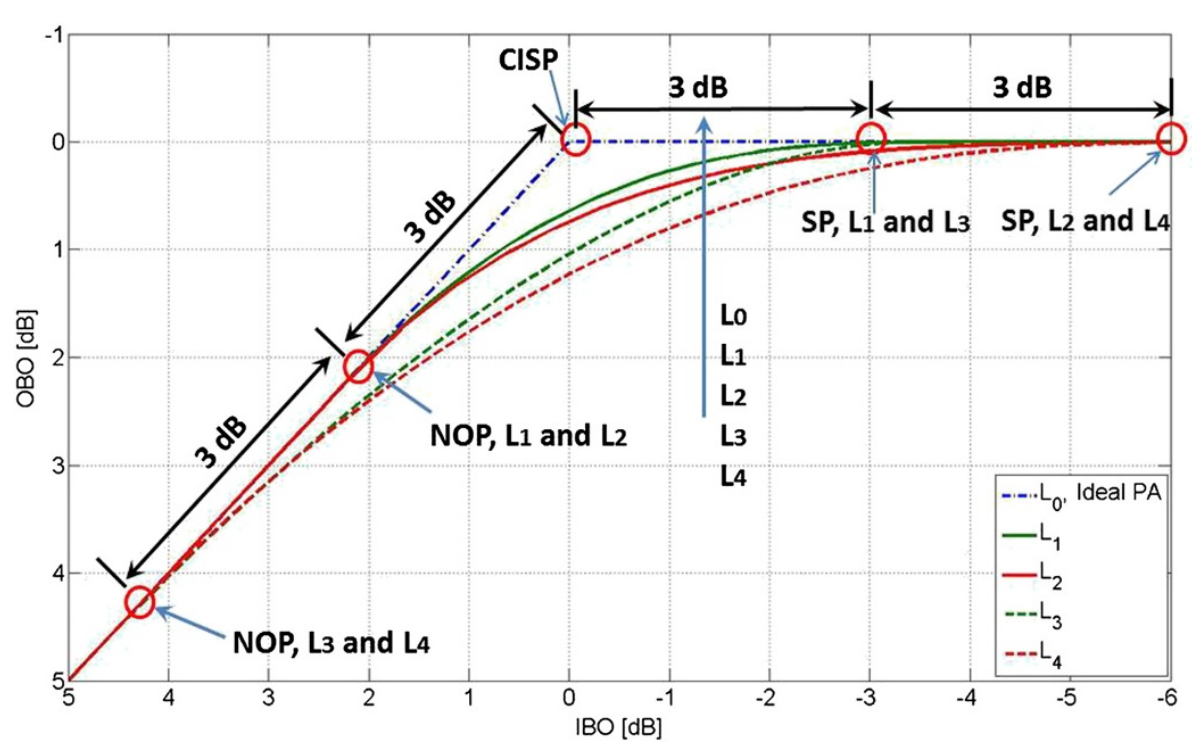

Figure 2 The envelope characters of five example SSPAs. These five example PAs have various combinations of NOP and SP.

and

$$
\begin{aligned}
\operatorname{PS}_{\mathrm{IMD}}\left(f, \sigma^{2}\right)= & \mathrm{DDF}_{3}(f) \cdot \mid \sum_{k=1}^{L} b_{k} \cdot\left[J_{1}\left(\frac{2 \pi k}{D_{\bmod }} \sqrt{\frac{\sigma^{2}}{N}}\right)\right]^{3} \\
& \times\left.\left[J_{0}\left(\frac{2 \pi k}{D_{\bmod }} \sqrt{\frac{\sigma^{2}}{N}}\right)\right]^{N-3}\right|^{2}
\end{aligned}
$$

where $f$ denotes the frequency; $\delta[$.$] is the Dirac function;$ $\mathrm{DDF}_{3}(f)$ denotes the distribution density function of third-order IMPs as defined in [15], and it can be easily counted according to the number of subcarriers. Here, IMD is represented by the dominated third-order IMPs, and higher-order IMPs are omitted.

The relation between $D_{\bmod }$ and $\sigma^{2}$ is discussed in [18]. As derived in [16], we can have that

$$
\begin{aligned}
\frac{2 \pi}{D_{\bmod }} \sqrt{\frac{\sigma^{2}}{N}} & <0.05 \text { and } 1 \approx J_{0}\left(\frac{2 \pi k}{D_{\bmod }} \sqrt{\frac{\sigma^{2}}{N}}\right) \\
& >J_{1}\left(\frac{2 \pi k}{D_{\bmod }} \sqrt{\frac{\sigma^{2}}{N}}\right)>0
\end{aligned}
$$

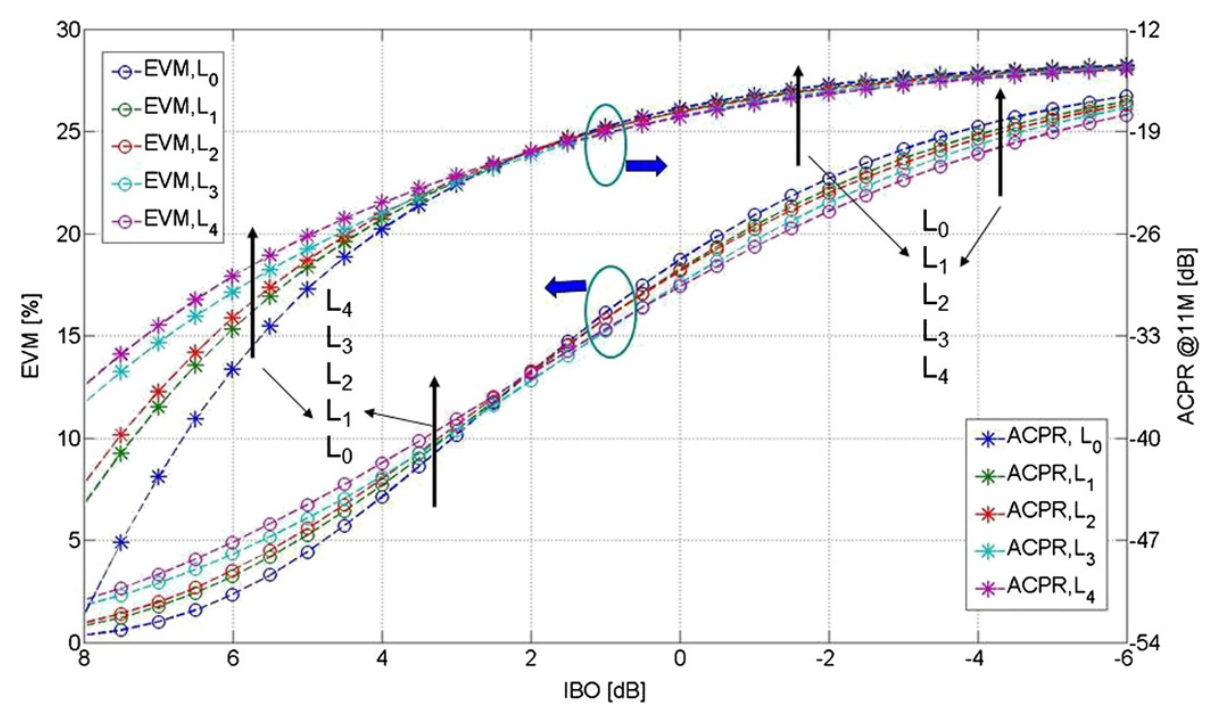

Figure 3 Simulation results of five example PAs. A 16QAM IEEE 802.11a signal is applied, and the ACPR graphs at 11 MHz frequency offset from center of the signal band of fundamental carriers. 
Applying (8), (6) and (7) can be re-written as

$$
\operatorname{PS}_{\text {fund }}\left(f, \sigma^{2}\right)=\left(\sum_{l=1}^{N} \delta\left(f-f_{l}\right)\right) \cdot\left|\sum_{k=1}^{L} b_{k} \cdot J_{1}\left(\frac{2 \pi k}{D_{\bmod }} \sqrt{\frac{\sigma^{2}}{N}}\right)\right|^{2}
$$

and

$$
\operatorname{PS}_{\mathrm{IMD}}\left(f, \sigma^{2}\right)=\operatorname{DDF}_{3}(f) \cdot\left|\sum_{k=1}^{L} b_{k} \cdot\left[J_{1}\left(\frac{2 \pi k}{D_{\text {mod }}} \sqrt{\frac{\sigma^{2}}{N}}\right)\right]^{3}\right|^{2}
$$

Thus, the signal-to-noise ratio (SNR) of nonlinearly amplified OFDM signal, $R_{\text {non }}\left(\sigma^{2}\right)$, can be written as

$$
\begin{aligned}
R_{\text {non }}\left(\sigma^{2}\right) & =\frac{\int_{\text {ch }} \operatorname{PS}_{\text {fund }}\left(f, \sigma^{2}\right) \cdot d f}{\int_{\operatorname{IMD}}\left(f, \sigma^{2}\right) \cdot d f} \\
& =\frac{N \cdot\left|\sum_{k=1}^{L} b_{k} \cdot J_{1}\left(\frac{2 \pi k}{D_{\bmod }} \sqrt{\frac{\sigma^{2}}{N}}\right)\right|^{2}}{\left[\int_{c h} \operatorname{DDF}_{3}(f) \cdot d f\right] \times\left|\sum_{k=1}^{L} b_{k} \cdot\left[J_{1}\left(\frac{2 \pi k}{D_{\text {mod }}} \sqrt{\frac{\sigma^{2}}{N}}\right)\right]^{3}\right|^{2}}
\end{aligned}
$$

where ch denotes the signal band of OFDM fundamental carriers. Based on the range estimation of Bessel series kernel in (7), i.e., $\pi \sqrt{\sigma^{2}} /\left(D_{\bmod } \sqrt{N}\right)$, it can be derived that

$$
\partial\left\{\left[J_{1}\left(\frac{2 \pi k}{D_{\text {mod }}} \sqrt{\frac{\sigma^{2}}{N}}\right)\right]^{3}\right\} /\left(\partial \sigma^{2} \partial k\right)>0
$$

This indicates that the power of IMD increased as input power as $\sigma^{2}$ increases, and the power of high-order IMD components are more sensitive to the variation of $\sigma^{2}$ than that of the low-order ones.

\section{Simulation observations and discussions}

In this section, the modulation fidelity of an IEEE 802.11a OFDM signal driving through five example SSPAs are presented. The EVM and ACPR are two specific measures of system SNR, which focuses on the in-band distortion and out-band spectrum regrowth, respectively. The EVM and ACPR results shown in this section are simulated based on the classical statistical (Stat) approach in [14]. The simulation results reveal how the PA nonlinearity is represented by the signal impairment over IBO and OBO domains, respectively, and the associated simulation observations are discussed based on above analysis.

In Figure 3, the simulated EVM and ACPR results of five example PAs are presented. It can be seen that when the input power is around linear or quasi-linear ranges (e.g., around $5 \mathrm{~dB}$ ), the order of nonlinear impairment of all five example PAs (from high to low) is $L_{0}, L_{1}, L_{2}, L_{3}$, and $L_{4}$. However, when the input power is close to saturation range (e.g., around $-4 \mathrm{~dB}$ ), the former order changes to $L_{4}, L_{3}, L_{2}, L_{1}$, and $L_{0}$. Referring to Table 1 , here we can notice that NOP has a very clear influence on system

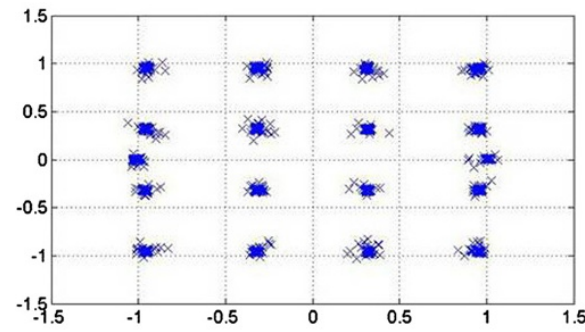

(a)

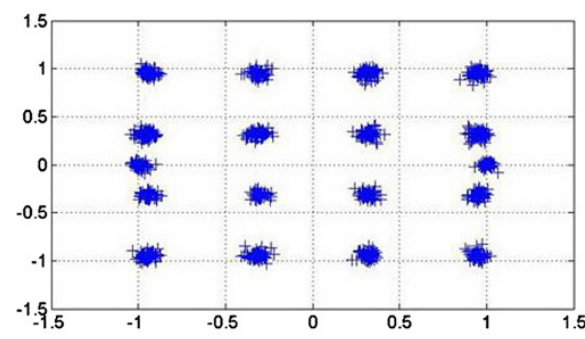

(c)

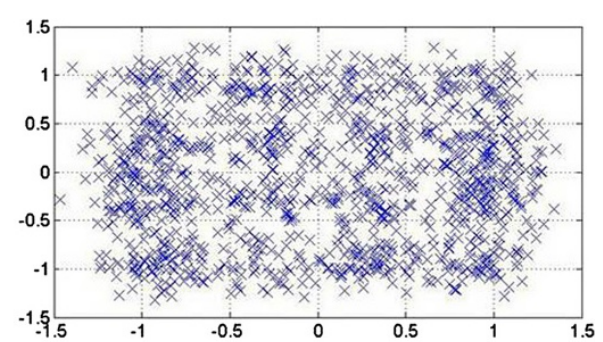

(b)

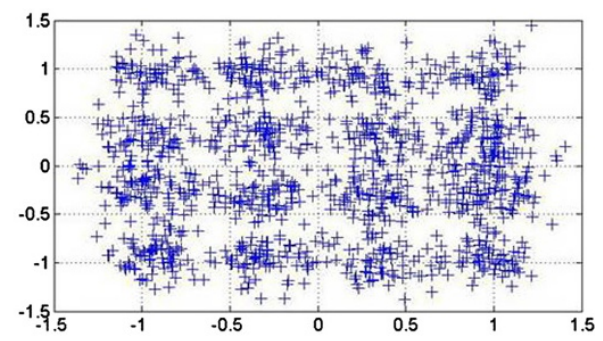

(d)

Figure 4 The constellation diagrams of $L_{0}$ and $L_{4}$. An 16QAM IEEE 802.11a OFDM signal is applied. (a,b) Output diagrams of $L_{0}$ at operating points of IBO 6 and $-2 \mathrm{~dB}$, respectively. (c,d) $L_{4}$ as the same configuration applied. 


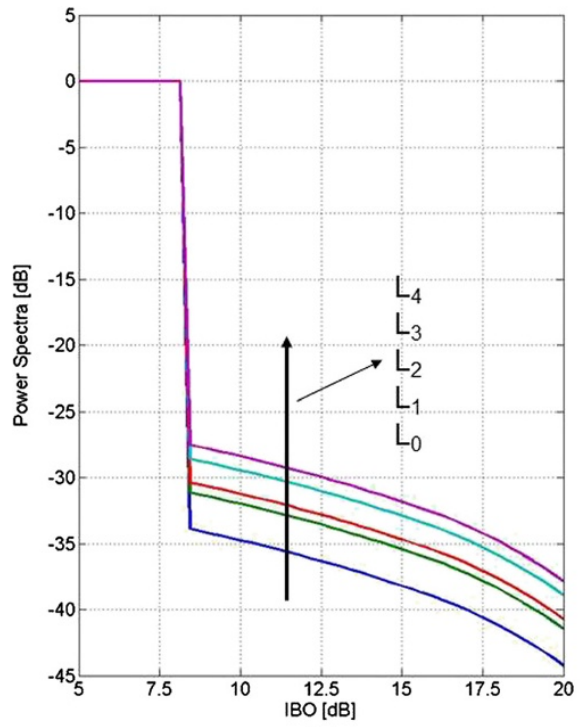

(a)

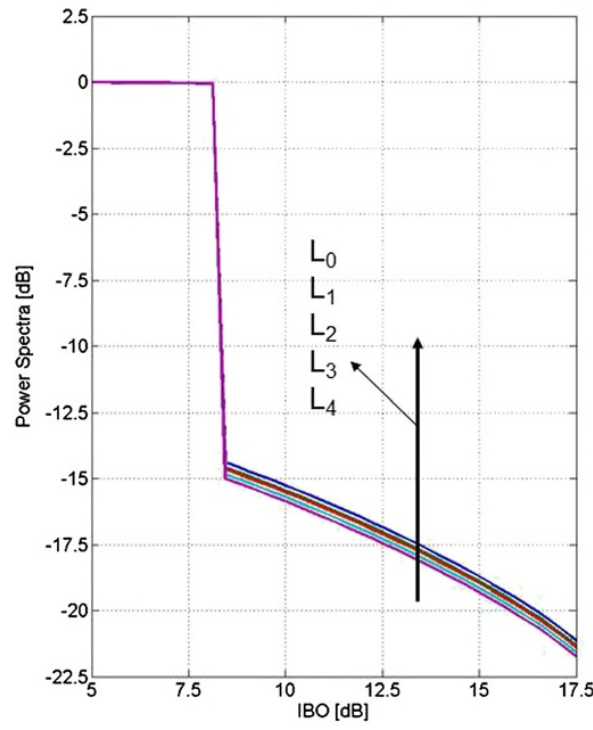

(b)

Figure 5 The power spectra of amplified OFDM signals. All example PAs are investigated as a 16QAM IEEE 802.11a signal applied. (a,b) Operation point of IBO 6 and $-2 \mathrm{~dB}$, respectively.

modulation fidelity and that the PAs have better linearity generate higher IMD as they are driven close to saturation ranges.

In Figure 4 , the constellation diagrams of amplified OFDM signals driven through $L_{0}$ and $L_{4}$ are presented, which are driven at operating points of IBO $5 \mathrm{~dB}$ and $-3 \mathrm{~dB}$, respectively. It can be seen that, from the constellation diagrams, $L_{4}$ generates higher IMD than $L_{0}$ does within low input power range, while $L_{0}$ generates higher
IMD than $L_{4}$ does within high input power range. This observation is consistent with that of Figure 3.

In Figure 5, the power spectrum graphs of all example PAs at IBO 6 and $-2 \mathrm{~dB}$ are presented. It can be seen that $L_{0}$ generates the lowest spectra regrowth than others do within low-input power range, while it generates the highest spectra regrowth within high-input power range. This observation is consistent with Figures 3 and 4, and it can be explained as follows. Since NOP has the clear

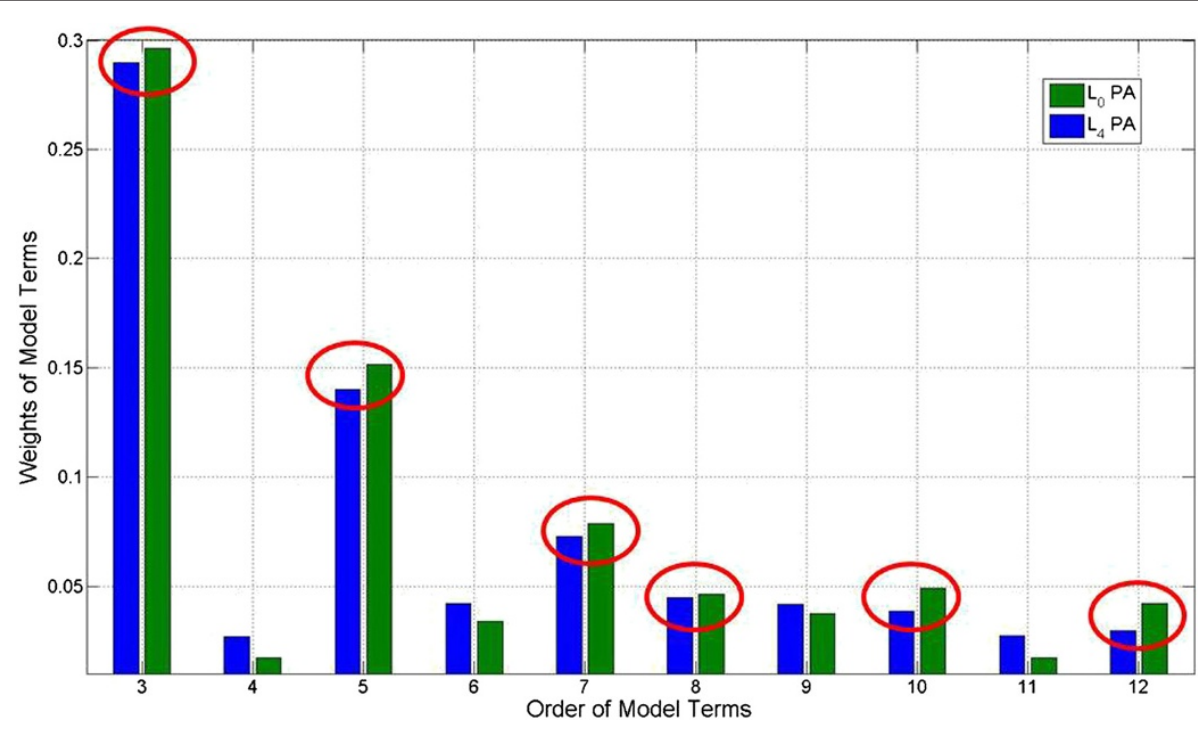

Figure 6 The weights of Bessel-Fourier model terms of example PA envelopes. The weights of model terms are normalized, and the high-order terms of $L_{0}$ is more significant than those of $L_{4}$. 


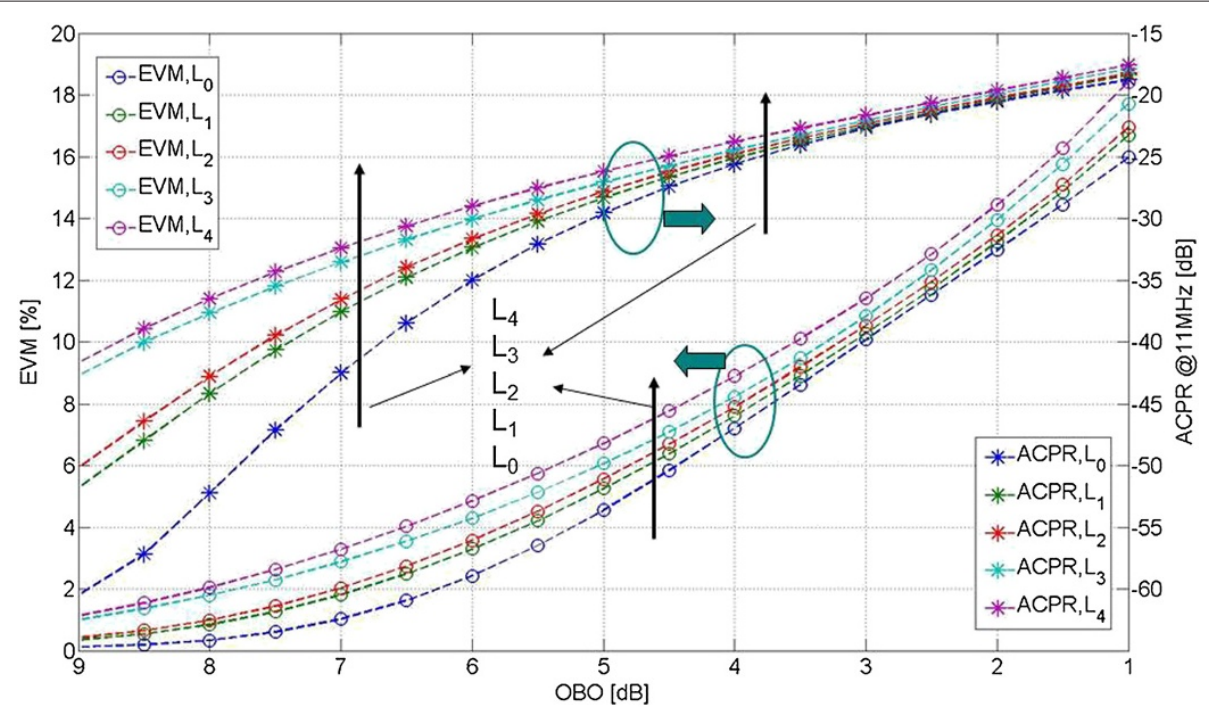

Figure 7 EVMs and ACPR results of all example PAs. The results are displayed over OBO domain, and a 16QAM IEEE 802.11a signal is applied.

influence on the convergence of coefficient spectrum of Bessel-Fourier PA model, and as shown in Figure 6 below, the weight of high-order model terms clearly increases as $D_{\text {NOP-CISP }}$ decreases. For example, the Bessel-Fourier PA model of $L_{0}$ has more high-order terms than that of $L_{4}$ does, since $L_{0}$ has a smaller $D_{\text {NOP-CISP }}$ than $L_{4}$ does. Furthermore, As analyzed around (12), the power of highorder IMD components is more sensitive to the variation of $\sigma^{2}$ than that of the low-order ones. That is, as $\sigma^{2}$ increases, the high-order model components show clear weights in total IMD. Therefore, IMD generated by $L_{0}$ is higher than that generated by $L_{4}$ as operating point is close to saturation points.

In Figure 6, the weights of Bessel-Fourier model terms of $L_{0}$ and $L_{4}$ example PAs are presented. It can be seen that as analyzed above, the weights of high-order terms of $L_{0}$ PA are higher than those of $L_{4}$. This is because $L_{0}$ has a relatively sharp variation at CISP. Thus, as observed in above figures, the modulation fidelity of $L_{0}$ shows more significant degradation than $L_{4}$ does when input power increased.

In Figure 7, the EVM and ACPR results are displayed over OBO domain. It can be seen here that the PAs having relatively higher linearity, such as $L_{0}$, show better modulation fidelity over the whole $\mathrm{OBO}$ range. This is because that $\mathrm{OBO}$ is not sensitive to IBO when PAs are driven close to saturation range.

\section{Conclusions}

In this paper, a comparison analysis is presented to investigate the system performance when both SP and nonlinearity onset point (NOP) variations are considered. The simulations show that how the PA envelope variation can be represented by the signal impairment over IBO and $\mathrm{OBO}$ domains, respectively. It is interesting to find that although SP attracts much concern in current studies, NOP actually has the clear influence on modulation fidelity of nonlinear OFDM transmitter systems. Moreover, it is observed when input power gets close to saturation range, the PAs having relatively higher nonlinearity show relatively lower distortion level over the input power domain. These interesting simulation observations above are analytically explained based on a mixed time domain and statistical analysis of the IMD mechanism.

\section{Competing interests}

The authors declare that they have no competing interests.

\section{Authors' information}

$Y L, M D$, and $Y J$ are from State Key Laboratory of Advanced Optical Communication Systems and Networks, School of Electronic Engineering and Computer Science, Peking University, Haidian District, Beijing, China.

\section{Acknowledgements}

The authors wish to acknowledge the support from State Key Laboratory of Advanced Optical Communication Systems and Networks, School of Electronic Engineering and Computer Science, Peking University. The authors also wish to acknowledge the suggestions from Prof. Máirtín O'Droma in the Telecommunications Research Centre, University of Limerick, Ireland.

Received: 9 January 2014 Accepted: 28 February 2014 Published: 3 April 2014

\section{References}

1. M O'Droma, I Ganchev, The creation of a ubiquitous consumer wireless world through strategic ITU-T standardization. IEEE Commun. Mag. 10, 158-165 (2010). doi:10.1109/MCOM.2010.5594691

2. H Yoo, F Guilloud, R Pyndiah, Amplitude PDF analysis of OFDM signal using probabilistic PAPR reduction method. EURASIP J. Wireless Commun. Netw (2011). doi:10.1155/2011/983915

3. Y Lei, M O'Droma, E Bertran, P Gilabert, On modeling of nonlinear distortion in OFDM transmitter systems, in Asia-Pacific Microwave Conference (Hong Kong, China, December 2008), pp. 16-20. doi:10.1109/APMC.2008.4958103 
4. T Helaly, R Dansereau, M El-Tanany, An efficient measure for nonlinear distortion severity due to HPA in downlink DS-CDMA signals. EURASIP J. Wireless Commun. Netw (2010). doi:10.1155/2010/945427

5. IEEE,Local and metropolitan area networks Specific requirements. Part 11: Wireless LAN Medium Access Control (MAC) and Physical Layer (PHY) Specifications. ANSI/IEEE Std 802.11a, (1999)

6. SV Zhidkov, Performance analysis of multi-carrier systems in the presence of smooth nonlinearity. EURASIP J. Wireless Commun. Netw (2004). doi:10.1155/S1687147204406124

7. AN D'Andrea, AN Lottici, R Reggiannini, Nonlinear predistortion of OFDM signals over frequency-selective fading channels. IEEE Trans. Commun. 5, 837-843 (2001). doi:10.1109/26.923807

8. R Singla, S Sharma, Digital predistortion of power amplifiers using look-up table method with memory effects for LTE wireless systems. EURASIP J. Wireless Commun. Netw (2012). doi:10.1186/1687-1499-2012-330

9. E Costa, S Pupolin, M-QAM-OFDM system performance in the presence of a nonlinear amplifier and phase noise. IEEE Trans. Commun. 3, 462-472 (2002). doi:10.1109/26.990908

10. M O'Droma, N Mgebrishvili, A Goacher, New percentage linearization measures of the degree of linearization of HPA nonlinearity. IEEE Commun. Lett. 4, 214-216 (2004). doi:10.1109/LCOMM.2004.823368

11. M O'Droma, N Mgebrishvili, On quantifying the benefits of SSPA linearization in UWC-136 systems. IEEE Trans. Sig. Process. 7, 2470-2476 (2005). doi:10.1109/TSP.2005.849190

12. D Dardari, V Tralli, A Vaccari, A theoretical characterization of nonlinear distortion effects in OFDM systems. IEEE Trans. Commun. 10, 1755-1764 (2000). doi:10.1109/26.871400

13. D Schreurs, M O'Droma, A Goacher, M Gardinger, RF Power Amplifier Behavioral Modelling. (Cambridge University Press, Cambridge, 2009)

14. M O'Droma, N Mgebrishvili, Signal modelling classes for linearized OFDM SSPA behavioral analysis. IEEE Commun. Lett. 2, 127-129 (2005). doi:10.1109/LCOMM.2005.02030

15. Y Lei, M O'Droma, Behavioural analysis of internal mechanism of nonlinear OFDM signals, in IEEE Global Telecommunication Conference (Hawaii, 30 November to 4 December 2009). doi:10.1109/GLOCOM.2009.5425495

16. Y Lei, M O'Droma, Y Jin, A practical analysis and performance optimization in OSTBC based nonlinear MIMO-OFDM systems. IEEE Trans. Commun. $\mathbf{3}$ 930-938 (2014). doi:10.1109/TCOMM.2014.010414.130533

17. M O'Droma, S Meza, Y Lei, New modified Saleh models for memoryles nonlinear power amplifier behavioural modelling. IEEE Commun. Lett. 12, 1007-1009 (2009). doi:10.1109/LCOMM.2009.12.0902222

18. M O'Droma, Y Lei, A new Bessel-Fourier memoryless nonlinear power amplifier behavioral model. IEEE Micro. Wirel. Comp. Lett. 1, 25-27 (2013). doi:10.1109/LMWC.2012.2236082

doi:10.1186/1687-1499-2014-52

Cite this article as: Lei et al: The sensitivity of modulation fidelity on PA envelope variation in OFDM transmitter systems. EURASIP Journal on Wireless Communications and Networking 2014 2014:52.

\section{Submit your manuscript to a SpringerOpen ${ }^{\circ}$ journal and benefit from:}

- Convenient online submission

- Rigorous peer review

- Immediate publication on acceptance

- Open access: articles freely available online

- High visibility within the field

- Retaining the copyright to your article

Submit your next manuscript at $\boldsymbol{~ s p r i n g e r o p e n . c o m ~}$ 